\title{
Multiconfiguration Molecular Mechanics Studies for the Potential Energy Surfaces of the Excited State Double Proton Transfer in the 1:1 7-Azaindole: $\mathrm{H}_{2} \mathrm{O}$ Complex
}

\author{
Jeong-A Han and Yongho Kim* \\ Department of Chemistry, Kyung Hee University, Yongin, Gyeonggi-do 446-701, Korea. ${ }^{*}$ E-mail: yhkim@khu.ac.kr \\ Received November 19, 2009, Accepted December 18, 2009
}

\begin{abstract}
The multiconfiguration molecular mechanics (MCMM) algorithm was used to generate potential and vibrationally adiabatic energy surfaces for excited-state tautomerization in the 1:1 7-azaindole: $\mathrm{H}_{2} \mathrm{O}$ complex. Electronic structures and energies for reactant, product, transition state were computed at the CIS/6-31G(d,p) level of theory. The potential and vibrationally adiabatic energies along the reaction coordinate were generated step by step by using 16 high-level Shepard points, which were computed at the CIS/6-31G(d,p) level. This study shows that the MCMM method was applied successfully to make quite reasonable potential and adiabatic energy curves for the excited-state double proton transfer reaction. No stable intermediates are present in the potential energy curve along the reaction coordinate of the excited-state double proton transfer in the 1:1 7-azaindole: $\mathrm{H}_{2} \mathrm{O}$ complex, indicating that these two protons are transferred concertedly. The change in the bond distances along the reaction coordinate shows that two protons move very asynchronously to make an $\mathrm{H}_{3} \mathrm{O}^{+}$-like moiety at the transition state.
\end{abstract}

Key Words: Excited-state tautomerization, MCMM, Potential energy surface

\section{Introduction}

Proton transfer is one of the primary processes involved in many chemical and biological reactions. Multiple proton transfer plays important role to numerous processes of biological importance including enzymatic reactions and transport phenomena in biological membranes. In particular, tautomerization through bridging hydrogen bonds in the 7-azaindole (7AI) system is an interesting biological model that has been studied extensively both experimentally and theoretically. Kasha et al. studied 7AI as a model for hydrogen bonding in DNA base pairs. ${ }^{1}$ 7AI has an $\mathrm{N}-\mathrm{H}$ bond and a heteroaromatic $\mathrm{N}$ atom as hydrogen bond donor and acceptor site, respectively, and the normal form becomes unstable with respect to the tautomer form in the $\mathrm{S}_{1}$ state. ${ }^{2,3}$ The monomer species of 7AI is capable of excited state double proton transfer (ESDPT) from the five-membered ring (donor site) to the six-membered ring (acceptor site) in a variety of environments, including a gas phase as well as solvent clusters when assisted by solvent molecules. Huang et $\mathrm{al}^{4}$ studied the 1:1 7AI:water complex in the first excited state in cold beams and reported a fluorescence lifetime of $8 \mathrm{~ns}$, which implies that the tautomerization rate constant of ESDPT cannot be larger than approximately $10^{7} \mathrm{~s}^{-1}$. However, no direct observation of ESDPT in this complex has yet been made.

In addition to the experimental observations in the gas phase, tautomerization in 7 $\mathrm{AI}$ has also been observed in the condensed phase in alcohol or water solutions. ${ }^{5-8}$ The tautomerization of 7AI in alcohols has been discussed in terms of a two step processes. ${ }^{9-12}$ The first step involves solvent reorganization to form a cyclically hydrogen-bonded 7AI-alcohol complex; the second step, intrinsic double proton transfer. Arrhenius activation energies of tautomerization were very similar to those associated with the solvent viscosity, ${ }^{11}$ which implies that solvent motion controls the reaction. If this motion was rate-limiting, no significant kinetic isotope effect (KIE) would be expected. However,
KIEs for excited-state tautomerization were observed in the 7AI complexes with various alcohols. ${ }^{6,911}$ Moogs et al. ${ }^{11}$ suggested that both solvent reorganization and the intrinsic proton transfer step determine the reaction rate, and Chen et al..$^{6}$ suggested that two protons are transferred concertedly in the intrinsic double proton transfer step based on the observed rates and KIEs satisfying the rule of geometric mean (RGM). However, the mechanism of ESDPT in water remains unclear. Chou et al. ${ }^{13}$ measured rates and KIEs for ESDPT of the 1:1 3-cyano-7AI: water complex to clarify the mechanism of 7AI in water. These authors and others ${ }^{5,10,13,14}$ suggested that a fast excited-state equilibrium between 1:1 cyclic hydrogen-bonded complex and randomly hydrated complexes is established and followed by proton transfer that may be governed by a tunneling mechanism. Chen et al. ${ }^{6}$ observed the breakdown of the RGM in water and suggested the stepwise ESDPT mechanism. However theoretical studies suggested the concerted mechanism. Chaban and co-workers ${ }^{2,3}$ studied the ground and excited state tautomerization processes in 7AI with and without mediating solvent using ab initio calculations. They used a CASSCF procedure with multireference second-order perturbation theory (MCQDPT2) to include dynamic electron correlation and calculate energetics and intrinsic reaction coordinates for the hydrogen transfer process in the ground and first excited states of the 7AI and the 7AI$\mathrm{H}_{2} \mathrm{O}$ complexes. They demonstrated that the dynamic electron correlation and additional water molecule dramatically reduced the activation barrier. Furthermore, they showed that the ESDPT in the 7AI- $\mathrm{H}_{2} \mathrm{O}$ complex occurs via a concerted process.

Very recently, Kina et al. ${ }^{15}$ performed ab initio molecular dynamics (AIMD) simulations for the 7AI- $\mathrm{H}_{2} \mathrm{O}$ complex; they showed that the ESDPT occurs at $t=\sim 50 \mathrm{fs}$ after the photoexcitation in the gas phase via a concerted and asynchronous process. They also presented the AIMD simulation including the surrounding water molecules by effective fragment potential and suggested that the ESDPT takes places asynchronously in 
both the gas phase and in solution. The mechanism was not altered by the solvent effect. In the trajectory of the AIMD simulations, the $\mathrm{O}-\mathrm{H}$ proton moves first to the 6-membered ring followed by movement of the $\mathrm{N}-\mathrm{H}$ proton from the 5-membered ring to water. However, the TS structures obtained by Chaban et al $^{2}$ and Kina et al. ${ }^{15}$ suggest that the N-H proton from the 5membered ring moves first, and the water proton follows. The AIMD trajectory was far from the intrinsic reaction coordinate that might be one of the high-energy reaction paths, thus further investigation is required. Asynchronous multiple proton transfers in other reactions have been reported previously. ${ }^{16-21}$

Tautomerization involves transfer of a light $(\mathrm{H})$ atom between heavy $(\mathrm{N}$ and $\mathrm{O}$ ) atoms, so quantum mechanical tunneling is expected to have a significant impact on the reaction rate. However, the HH/DD KIEs in aqueous solution ${ }^{5,6}$ do not provide a clear evidence for significant tunneling. To interpret these KIE values correctly, a global potential energy surfaces for the excited state and a dynamics theory that can handle multidimensional tunneling in large molecular systems are required. An accurate estimate of the potential energy surface is required to investigate the asynchronicity of double proton transfer and reaction dynamics in detail, but these are extremely difficult for excited state reactions. Therefore, theoretical studies of excited-state reaction dynamics, including quantum mechanical tunneling, are very rare. Recently multiconfiguration molecular mechanics (MCMM) has been introduced to generate multidimensional potential energy surface. ${ }^{22}$ This algorithm can be regarded as a dual-level scheme that uses molecular mechanics potential functions as the lower level and electronic structure theory as the higher level. This method was applied well to the proton-transfer reactions in hydrogen bonded systems since these reactions can be expressed well by a double-well potential function. ${ }^{23}$ In this study, we will show that the potential energy surface of the ESDPT in the 1:1 7AI:water complex can be generated reasonably well by using the MCMM algorithm. One can calculate rate constants by the canonical variational transition state theory including tunneling approximation, and the quality of rate constants depend on the level of quantum mechanical theory.

\section{Computational Methods}

Details of the multiconfiguration molecular mechanics (MCMM) algorithm have been described elsewhere in detail; ${ }^{22}$ therefore, only a brief description of each method employed will be given here. In the MCMM formalism, the reactive system can be defined by using several valence bond configurations or, more generally, diabatic configurations corresponding to each of the minima on the potential energy surface. Each configuration can be described by a molecular mechanics potential, $V_{11}(\mathbf{q})$ and $V_{22}(\mathbf{q})$, which is correct in the vicinity of the well. For a geometry $\mathbf{q}$ far from the minima the energy can be expressed in terms of the two diabatic configurations by solving the secular equation:

$$
\left|\begin{array}{cc}
V_{11}-V & V_{12} \\
V_{12} & V_{22}-V
\end{array}\right|=0
$$

where the term $V_{12}(\mathbf{q})$ is called the resonance energy function or resonance integral, and $V$ denotes the lowest-energy eigenvalue of $\mathbf{V}$. The element $V_{i j}$ of the matrix $\mathbf{V}$ may be considered to be the representation of the electronic Hamiltonian (including nuclear repulsion) in an electronically diabatic basis, and the eigenvalue $V$ is the expectation value of the electronic Hamiltonian (including nuclear repulsion) for the lowest-energy electronically adiabatic state. This eigenvalue is given by

$$
\begin{aligned}
V(\mathbf{q})= & \frac{1}{2}\left\{\left(V_{11}(\mathbf{q})+V_{22}(\mathbf{q})\right)\right. \\
& \left.-\left[\left(V_{11}(\mathbf{q})-V_{22}(\mathbf{q})\right)^{2}+4 V_{12}(\mathbf{q})^{2}\right]^{1 / 2}\right\}
\end{aligned}
$$

Note that $V$ tends to the energy $V_{11}$ of configuration 1 (reactants) or the energy $V_{22}$ of configuration 2 (products) whenever the value of the resonance integral is negligible. The critical issue in the MCMM formulation is the calculation of that resonance integral and its derivatives, since the $V_{11}$ and $V_{22}$ terms and their derivatives are extracted from the molecular mechanics force field. Note that $V_{11}$ is generated using the connectivity (valence structure) of reactants, and $V_{22}$ is generated using the connectivity of products.

From Eq. (1), $V_{12}(\mathbf{q})$ can be expressed as:

$$
V_{12}(\mathbf{q})^{2}=\left[V_{11}(\mathbf{q})-V(\mathbf{q})\right]\left[V_{22}(\mathbf{q})-V(\mathbf{q})\right]
$$

Near the arbitrary geometry $\mathbf{q}^{(k)}$, each quantity on the right hand side of Eq. (3) can be expanded in Taylor's series. Thus,

$$
V(\mathbf{q} ; k) \cong V^{(k)}+\mathbf{g}^{(k)^{\top}} \cdot \Delta \mathbf{q}^{(k)}+\frac{1}{2} \Delta \mathbf{q}^{(k)^{\top}} \cdot \mathbf{f}^{(k)} \cdot \Delta \mathbf{q}^{(k)}
$$

where

$$
\Delta \mathbf{q}^{(k)}=\mathbf{q}-\mathbf{q}^{(k)}
$$

and $V^{(k)}, \mathbf{g}_{n}^{(k)}$, and $\mathbf{f}^{(k)}$ are the target energy, gradient, and Hessian matrix respectively of the reference point (note that if the reference geometry corresponds to a saddle point or local minimum (well) on the potential energy hypersurface, $\mathbf{g}_{n}^{(k)}$ is zero). Furthermore we expand the diagonal elements of $V_{n n}$ around the geometry $\mathbf{q}^{(k)}$ :

$$
V_{n n}(\mathbf{q} ; k) \cong V_{n}^{(k)}+\mathbf{g}_{n}^{(k)^{\top}} \Delta \mathbf{q}+\frac{1}{2} \Delta \mathbf{q}^{\top} \mathbf{f}_{n}^{(k)} \Delta \mathbf{q}
$$

where

$$
V_{n}^{(k)}=V_{n n}\left(\mathbf{q}^{(k)}\right), \mathbf{g}_{n}^{(k)}=\left(\frac{\partial V_{n n}}{\partial \mathbf{q}}\right)_{\mathbf{q}=\mathbf{q}^{(k)}}, \mathbf{f}_{n}^{(k)}=\left(\frac{\partial^{2} V_{n n}}{\partial \mathbf{q} \partial \mathbf{q}}\right)_{\mathbf{q}=\mathbf{q}^{(k)}}
$$

for $n=1,2$. Note that the quantities with superscript $(k)$ are constants evaluated at the geometry $\mathbf{q}^{(k)}$ of each reference point, $k$, and therefore are independent of $\mathbf{q}$. The quantities $V_{n n}$ defined 
in Eq. (6) and $V_{12}(\mathbf{q} ; k)$ and its derivatives are functions dependent on the geometry, $\mathbf{q}$, as well as on the geometry of the reference point $k$. Substituting Eqs. (4) and (6) into (3), we obtain the following general form of the $V_{12}$ term:

$$
\begin{aligned}
V_{12}(\mathbf{q} ; k)^{2} \cong\left(V_{1}^{(k)}-V^{(k)}\right)\left(V_{2}^{(k)}-V^{(k)}\right) \\
+\left(V_{2}^{(k)}-V^{(k)}\right)\left(\mathbf{g}_{1}^{(k)}-\mathbf{g}^{(k)}\right)^{\top} \Delta \mathbf{q} \\
+\left(V_{1}^{(k)}-V^{(k)}\right)\left(\mathbf{g}_{2}^{(k)}-\mathbf{g}^{(k)}\right)^{\top} \Delta \mathbf{q} \\
+\frac{1}{2}\left(V_{2}^{(k)}-V^{(k)}\right) \Delta \mathbf{q}^{\top}\left(\mathbf{f}_{1}^{(k)}-\mathbf{f}^{(k)}\right) \Delta \mathbf{q} \\
+\frac{1}{2}\left(V_{1}^{(k)}-V^{(k)}\right) \Delta \mathbf{q}^{\top}\left(\mathbf{f}_{2}^{(k)}-\mathbf{f}^{(k)}\right) \Delta \mathbf{q} \\
+\left[\left(\mathbf{g}_{1}^{(k)}-\mathbf{g}^{(k)}\right)^{\top} \Delta \mathbf{q}\right]\left[\left(\mathbf{g}_{2}^{(k)}-\mathbf{g}^{(k)}\right)^{\top} \Delta \mathbf{q}\right] .
\end{aligned}
$$

Equation (8) provides an analytic expression for evaluating the resonance integral in the vicinity of a reference point. However, when dealing with a nuclear configuration far from the reference point $k$, the value $V_{12}(\mathbf{q} ; k)$ given by Eq. (8) is unbounded either positive or negative, and hence the value of $V(\mathbf{q})$ given by Eq. (2) diverges. In this paper, we applied a modified version of a Shepard interpolation scheme ${ }^{24,25}$ previously applied directly to $V(\mathbf{q})$. This method does not make any assumption, and it allows for systematic improvement as the number $M$ of points $k$ is increased.

The Shepard interpolation algorithm, in internal coordinates q, yields

$$
V_{12}^{S}(\mathbf{q})=\sum_{k=1}^{M} W_{k}(\mathbf{q}) V_{12}{ }^{\prime}(\mathbf{q} ; k)
$$

where the normalized weights $W_{k}(\mathbf{q})$ are defined as

$$
W_{k}(\mathbf{q})=\frac{w_{k}(\mathbf{q})}{w(\mathbf{q})}
$$

and

$$
w(\mathbf{q})=\sum_{l=1}^{M} w_{l}(\mathbf{q})
$$

and $V_{12}{ }^{\prime}(\mathbf{q} ; k)$ is a modified quadratic function

$$
\left[V_{12}{ }^{\prime}(\mathbf{q} ; k)\right]^{2}=\left[V_{12}(\mathbf{q} ; k)\right]^{2} u(\mathbf{q} ; k)
$$

where the quadratic part is

$$
\begin{aligned}
& {\left[V_{12}(\mathbf{q} ; k)\right]^{2}} \\
& =D^{(k)}\left[1+\mathbf{b}^{(k)^{\top}}\left(\mathbf{q}-\mathbf{q}^{(k)}\right)+\frac{1}{2}\left(\mathbf{q}-\mathbf{q}^{(k)}\right)^{\top} \mathbf{C}^{(k)}\left(\mathbf{q}-\mathbf{q}^{(k)}\right)\right]
\end{aligned}
$$

and the modification is

$$
u(\mathbf{q} ; k)=\left\{\begin{array}{ll}
\exp \left(-\delta /\left[V_{12}(\mathbf{q} ; k)\right]^{2}\right) & {\left[V_{12}(\mathbf{q} ; k)\right]^{2}>0} \\
0 & {\left[V_{12}(\mathbf{q} ; k)\right]^{2} \leq 0}
\end{array} .\right.
$$

We use a very small value of $\delta, 1 \times 10^{-8} E_{\mathrm{h}}{ }^{2}$ (where $1 E_{\mathrm{h}}=1$ hartree), so that Eq. (14) converges rapidly to 1 with increasing values of $\left[V_{12}(\mathbf{q} ; k)\right]^{2}$. The constants $D^{(k)}, \mathbf{b}^{(k)}$, and $\mathbf{C}^{(k)}$ are chosen such that Eq. (12) combined with Eq. (2) reproduces the expansion. The weighting function we are using is

$$
w_{k}(\mathbf{q})=\frac{\left[d_{k}(\mathbf{q})\right]^{-4}}{\sum_{i=1}^{M} \frac{1}{\left[d_{k}(\mathbf{q})\right]^{4}}}
$$

where $d_{k}(\mathbf{q})$ denotes a generalized distance between $\mathbf{q}$ and $\mathbf{q}(k)$ defined as:

$$
d_{k}(\mathbf{q})=\sqrt{\sum_{i=1}^{N^{\prime}}\left(q_{i}-q_{i}^{(k)}\right)^{2}}
$$

where $N$ is less than or equal to the number $N$ of internal coordinates used in Eqs. (2)-(10).

The geometries of the reactant complex, product complex and TS in the excited electronic state were optimized at the CIS/6$31 \mathrm{G}(\mathrm{d}, \mathrm{p})$ level using the GAUSSIAN03 quantum mechanical package. ${ }^{26}$ The potential energies along the reaction coordinate were generated step by step by using 16 high-level Shepard points, which were computed at the CIS/6-31G $(\mathrm{d}, \mathrm{p})$ level. The minimum energy path (MEP) was obtained in the mass-scaled coordinate by $1 \mathrm{amu}$. The MC-TINKERATE ${ }^{34}$ that interconnects POLYRATE $^{35}$ and MC-TINKER ${ }^{36}$ program packages was used for the reaction coordinate calculations.

\section{Results and Discussion}

The optimized geometries for the reactant, product, and the transition state of the excited state at the CIS level using the 6$31 \mathrm{G}(\mathrm{d}, \mathrm{p})$ and $6-31+\mathrm{G}(\mathrm{d}, \mathrm{p})$ basis sets were illustrated in Fig. 1. At the $6-31 \mathrm{G}(\mathrm{d}, \mathrm{p})$ level, the $\mathrm{O}_{16}-\mathrm{H}_{18}$ distance at the TS is $1.09 \AA$, which is only $0.14 \AA$ longer than its value at the reactant, whereas the $\mathrm{N}_{1}-\mathrm{H}_{10}$ bond distance is increased by $0.33 \AA$. The $\mathrm{H}_{10}$ moved more than half-way from $\mathrm{N}_{1}$ toward $\mathrm{O}_{16}$ atoms but the $\mathrm{H}_{18}$ atom moved very little, which generates a $\mathrm{H}_{3} \mathrm{O}^{+}$-like moiety at the TS. The $\mathrm{O}_{16}-\mathrm{H}_{18}$ distance of TS was nearly the same as that at the CASSCF(10,9)/DZP level, ${ }^{2}$ but the $\mathrm{O}_{16}-\mathrm{H}_{10}$ distances was about $0.06 \AA$ shorter. These results mean that the computed TS at the CIS level has stronger $\mathrm{H}_{3} \mathrm{O}^{+}$-like character compared with that at the CASSCF level, which would generate larger ion-pair property in the TS. Chaban and Gordon ${ }^{2}$ reported the TS structure in the ground state, where the $\mathrm{H}_{18}$ atom moved all the way to the $\mathrm{N}_{7}$ atom but the $\mathrm{H}_{10}$ moved only $0.14 \AA$, which makes a OH-like moiety. These results suggest that 7-AI is more basic and acidic than water in the ground and excitedstate, respectively, so the $\mathrm{N}_{1}-\mathrm{H}_{10}$ bond in the excited-state becomes weak to be broken easily.

Barrier heights and reaction energies depending on theore- 

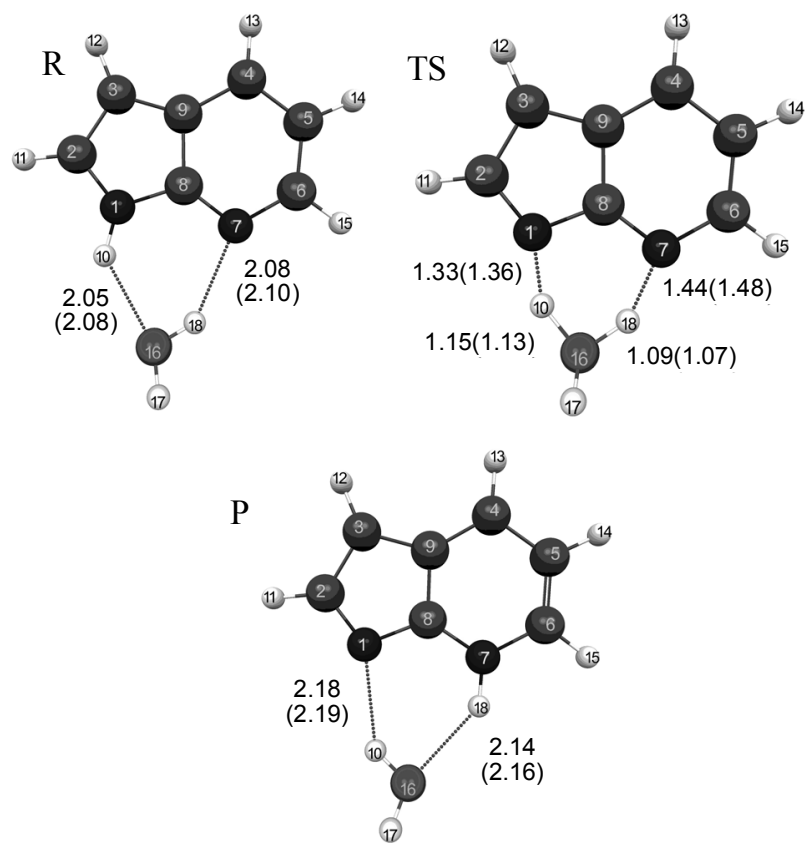

Figure 1. Selected bond distances in $\AA$ optimized at the CIS/6-31G(d,p) and $\mathrm{CIS} / 6-31+\mathrm{G}(\mathrm{d}, \mathrm{p})$ level (numbers in parenthesis) for Reactant, Transition State, and Product in the excited state.

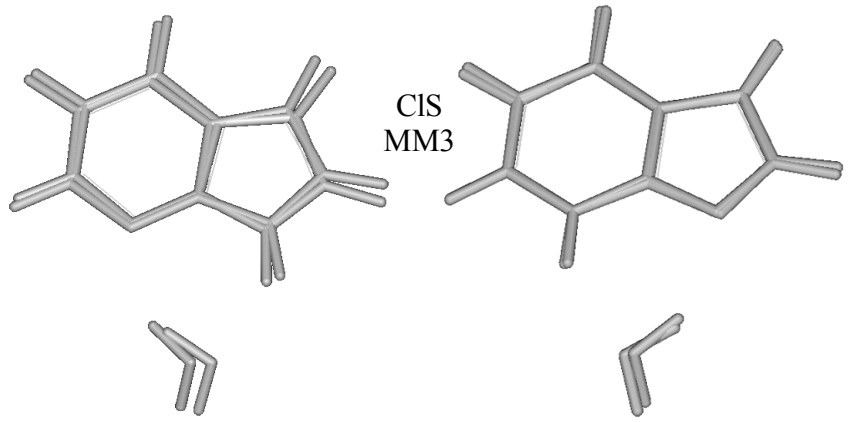

Figure 2. The superimposed structures of reactant and product optimized at the CIS/6-31G(d,p) and MM3 levels. tical levels are given in Table 1 . In the ground state, the normal form $(\mathrm{R})$ is lower in energy than the tautomer form $(\mathrm{P})$, whereas, in the excited state, the normal form is higher in energy than the tautomer. Adding the diffuse functions on heavy atoms increased the barrier height both in the ground state and the excited state. Chaban and Gordon ${ }^{2}$ calculated the barrier heights at the CASSCF(10,9)/DZP and MCQDPT2 level, which were 18.20 and $9.80 \mathrm{kcal} / \mathrm{mol}$, respectively. They found that a water molecule bridging the nitrogen atoms in 7-AI greatly reduced the barrier. Time-dependent density functional theory slightly underestimates the barrier height in the excited state. The CIS and CASSCF methods tend to overestimate the energy barriers of the excited state, so the dynamic electron correlation should be included.

The main purpose in this paper is to show the applicability of the MCMM algorithm in the excited-state reactions rather than to make a highly accurate potential energy surface, so the CIS/6-31G(d,p) level would be good enough as the higher level in the MCMM. We have used the MM3 force field for the $V_{11}$ and $V_{22}$ terms in Eq. (1), and defined several force field parameters for 7 -azaindole that are missing. The new and modified parameters are listed in supplementary information. TINKER

Table 2. Geometric parameters in $\AA$ for reactant and product optimized at the CIS/6-31G(d,p) and modified MM3 levels

\begin{tabular}{lccccc}
\hline & \multicolumn{2}{c}{ Reactant } & & \multicolumn{2}{c}{ Product } \\
\cline { 2 - 3 } \cline { 5 - 6 } & CIS & MM3 & & CIS & MM3 \\
\hline $\mathrm{N}_{1}-\mathrm{C}_{2}$ & 1.331 & 1.339 & & 1.305 & 1.316 \\
$\mathrm{~N}_{1}-\mathrm{C}_{8}$ & 1.389 & 1.389 & & 1.377 & 1.385 \\
$\mathrm{~N}_{7}-\mathrm{C}_{8}$ & 1.301 & 1.306 & & 1.340 & 1.348 \\
$\mathrm{C}_{6}-\mathrm{N}_{7}$ & 1.392 & 1.399 & & 1.401 & 1.397 \\
$\mathrm{C}_{8}-\mathrm{C}_{9}$ & 1.427 & 1.426 & & 1.392 & 1.430 \\
$\mathrm{~N}_{1}-\mathrm{H}_{10}$ & 1.000 & 1.021 & & 2.179 & 2.339 \\
$\mathrm{O}_{16}-\mathrm{H}_{10}$ & 2.049 & 2.028 & & 0.949 & 0.952 \\
$\mathrm{O}_{16}-\mathrm{H}_{18}$ & 0.954 & 0.952 & & 2.136 & 2.057 \\
$\mathrm{~N}_{7}-\mathrm{H}_{18}$ & 2.075 & 2.214 & & 0.997 & 1.001 \\
\hline
\end{tabular}

Table 1. Relative energies with respect to reactant in the ground and excited state and imaginary frequencies of the transition state for the double proton transfer

\begin{tabular}{lccc}
\hline Computational Method & $\Delta V^{*}(\mathrm{kcal} / \mathrm{mol})$ & $\Delta E(\mathrm{kcal} / \mathrm{mol})$ & Freq $\left(\mathrm{cm}^{-1}\right)$ \\
\hline & Ground State & & \\
B3LYP/6-31G(d,p) & 20.8 & 9.35 & $1541 \mathrm{i}$ \\
B3LYP/6-31+G(d,p) & 22.1 & 9.41 & $1456 \mathrm{i}$ \\
MP2/6-31G(d,p) & 22.8 & 10.5 & $1507 \mathrm{i}$ \\
MP2/6-31+G(d,p) & 23.9 & 10.3 & $1591 \mathrm{i}$ \\
& Excited State & & $1724 \mathrm{i}$ \\
CIS/6-31G(d,p) & 25.2 & -17.7 & $1523 \mathrm{i}$ \\
CIS/6-31+G(d,p) & 27.2 & -15.6 & \\
TD-B3LYP/6-31G(d,p)//CIS/6-31G(d,p) & 5.34 & -20.6 & \\
TD-B3LYP/6-31+G(d,p)//CIS/6-31G(d,p) & 8.34 & -19.3 & $1773 \mathrm{i}$ \\
CASSCF(10,9)/6-31G(d,p)//CIS/6-31G(d,p) & 19.2 & -36.5 & \\
CASSCF(10,9)/DZP & 18.2 & -31.8 & \\
MCQDPT2//CASSCF(10,9)/DZP & 9.8 & -18.0 & \\
\hline
\end{tabular}




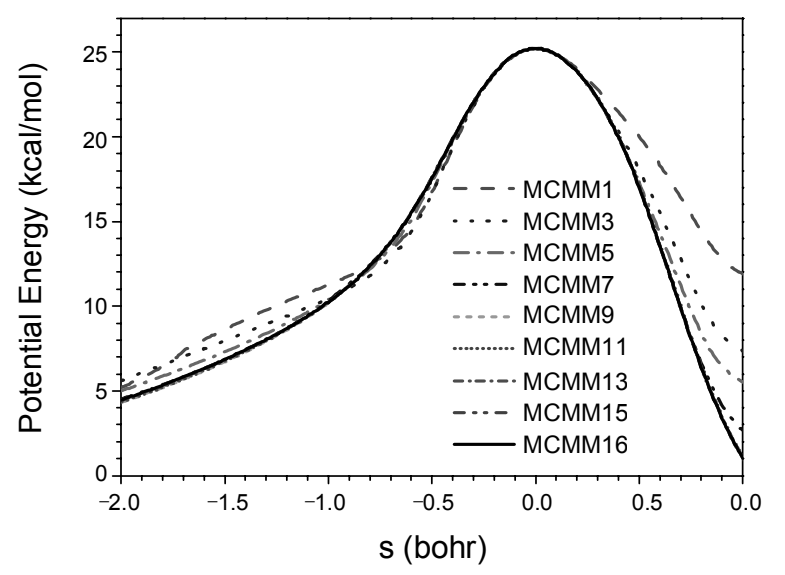

Figure 3. The potential energy curves along the minimum energy path calculated with the MCMM algorithm. The MCMM $n$ denotes the potential energy curve using $n$ high-level Shepard points.

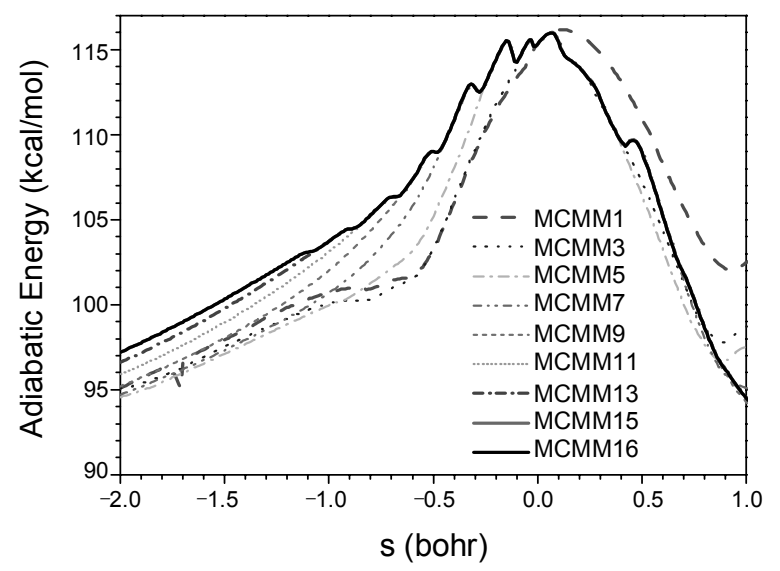

Figure 4. The vibrationally adiabatic energy curves along the minimum energy path calculated with the MCMM algorithm. The MCMM $n$ denotes the adiabatic energy curve using $n$ high-level Shepard points.

uses a variable-electronegativity self-consistent field (VESCF) treatment for conjugated $\pi$ systems. The VESCF treatment is used to reoptimize the parameters for $\mathrm{C}$ atoms in the conjugated system. However, once the new set of parameters for a given geometry is calculated using the VESCF method, there is no consistent procedure for calculating the gradients and Hessians. If we calculate the derivatives numerically, the parameters will be reoptimized for each geometry, and even though the changes in the geometry are small, they are large enough so that the numerical and analytical derivatives are different. The way we fixed this problem was to redefine $\mathrm{C}$ atoms in conjugated $\pi$ systems to a new atom type so that the VESCF calculation was not to be used. We defined an atom-type 20 for the $\mathrm{C}$ atoms in conjugated $\pi$ systems. The structures for reactant and product optimized at the CIS and MM3 levels are depicted in Fig. 2, and geometric parameters are in Table 2. Hydrogen bond distances of $\mathrm{N}_{7}-\mathrm{H}_{18}$ for reactant, and $\mathrm{N}_{1}-\mathrm{H}_{10}$ for product were predicted slightly longer at the modified MM3 level than those at the CIS/6-61G $(\mathrm{d}, \mathrm{p})$ level. In general the structures optimized using the modified MM3 parameters agree reasonably

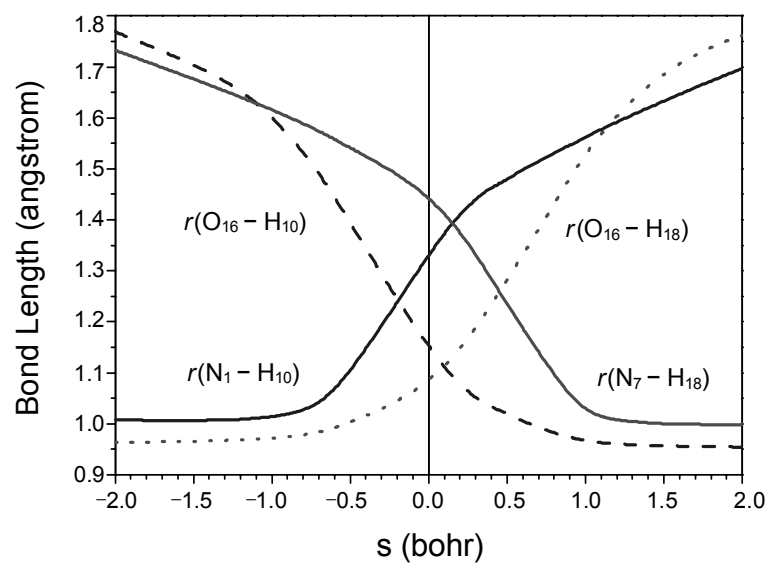

Figure 5. Bond distances in angstrom along the reaction coordinate for the double proton transfer of $7 \mathrm{AI}-\mathrm{H}_{2} \mathrm{O}$ complex.

well with those at the CIS level.

The potential energies along the reaction coordinate are shown in Fig. 3. We have used 16 high-level Shepard points step by step to generate the final potential energy surface. The potential energy curve interpolated with only one high-level point, which is for the transition state, is shown by the dashed line. We chose two additional structures for the high-level points from the MEP of this surface using one high-level point. In the previous MCMM studies, ${ }^{22}$ the additional structures are chosen at the point $1 / 2$ or $1 / 4$ down from the top of the barrier in terms of energy. This method does not work in this study since it does not consider the shape of the existing surface. The MEP at the point $1 / 2$ or $1 / 4$ down from the top would be far from the MEP of the true surface, since this part of the surface is influenced more by the MM3 level of theory. These inaccurate points would be used continuously as reference points for the following MCMM calculations, which makes unavoidable errors in the final potential energy surfaces. Therefore one should analyze the shape of the potential surface carefully to choose good structures for the high-level points.

We selected two structures at $\mathrm{s}=-0.005$ and $0.2 \mathrm{bohr}$ in the reactant and product side, respectively, from the interpolated surface using the single high-level point, as shown in Fig. 3. We have calculated energies, gradients, and Hessian using these structures at the CIS/6-31G(d,p) level, and added this information to generate a new potential energy surface. The dotted line in Fig. 3, which is denoted by MCMM3, is the potential curve using three high-level points. Two additional structures at $\mathrm{s}=-0.2$ and 0.4 are selected from the MEP of this potential surfaces, and energies, gradients, and Hessian are calculated for the additional high-level points. A new potential surface is generated by the interpolation with these two additional points, and this procedure is repeated until 16 high-level points are added. The final 16 points are at $\mathrm{s}=0.0,-0.005, \pm 0.2, \pm 0.4, \pm 0.6$, $\pm 0.8, \pm 1.0, \pm 1.2,+1.4$, and +1.6 bohr. As one can see in Fig. 3, the interpolated potential energy curves started converging to the final potential surface after 9 points were added (MCMM9). No stable intermediates are present in the potential energy curve along the reaction coordinate of the ESDPT, indicating that these two protons are transferred concertedly. 
Vibrationally adiabatic ground state energies, which are the sum of the classical potential energy and the zero-point energies, are shown in Fig. 4. As we added more high-level points for the interpolation, the adiabatic energy curves in the product side converged quite rapidly, but not in the reactant side. The reactant-side adiabatic energy curves were converged after 15 points were added. The interpolation seems to generate quite reasonable adiabatic energy curves, although there are slight winds between points near the top of the barrier. This means that the gradients and Hessian at the MM3 level are not good enough to predict the correct frequencies by the interpolation. The frequency seems more sensitive to this deficiency of the MM3 force field parameters than the potential energy.

The change in some bond distances along the reaction coordinate are shown in Fig. 5, where the bond distances of $\mathrm{N}_{1}-\mathrm{H}_{10}$, $\mathrm{O}_{16}-\mathrm{H}_{10}, \mathrm{O}_{16}-\mathrm{H}_{18}$, and $\mathrm{N}_{7}-\mathrm{H}_{18}$ are presented. As the reaction proceeds from the reactant to product, the $\mathrm{H}_{10}$ atom starts moving rapidly at about $\mathrm{s}=-0.7$ bohr from $\mathrm{N}_{1}$ in the 5-membered ring to $\mathrm{O}_{16}$ and the $r\left(\mathrm{~N}_{1}-\mathrm{H}_{10}\right)$ and $r\left(\mathrm{O}_{10}-\mathrm{H}_{10}\right)$ values are crossed at about $\mathrm{s}=-0.2$, whereas the $\mathrm{H}_{18}$ atom moves rather slowly from $\mathrm{O}_{16}$ to $\mathrm{N}_{7}$ in the 6-membered ring and the $r\left(\mathrm{O}_{16}-\mathrm{H}_{18}\right)$ and $r\left(\mathrm{~N}_{7-}\right.$ $\mathrm{H}_{18}$ ) values are crossed at about $\mathrm{s}=0.5 \mathrm{bohr}$. The two protons in flight move very asynchronously. Kina et al. ${ }^{15}$ carried out $a b$ initio MD simulations for the ESDPT in the 1:1 7-azaindole: water complex and reported that the two protons were transferred asynchronously and concertedly. Their results are consistent with this study, except that the order of transferring protons is opposite. These authors reported that the $\mathrm{H}_{18}$ atom first moves from $\mathrm{O}_{16}$ to $\mathrm{N}_{7}$, followed by the $\mathrm{H}_{10}$ atom from $\mathrm{N}_{1}$ to $\mathrm{O}_{16}$. As a result, an $\mathrm{OH}$-like moiety is formed at about $50 \mathrm{fs}$ of the $a b$ initio MD trajectory as presented in ref. 15 , which is inconsistent with the fact that 7AI becomes acidic in the $S_{1}$ state upon $\pi-\pi^{*}$ electronic excitation, ${ }^{37}$ and other high level electronic structure calculations. $^{2}$ This study shows that the ESDPT in the 1:1 7azaindole:water complex occurs asynchronously to make an $\mathrm{H}_{3} \mathrm{O}^{+}$-like moiety at the TS.

\section{Conclusion}

The MCMM algorithm was used to generate potential energy surfaces of the ESDPT in the 1:1 7-azaindole:water complex. High-level Shepard points calculated at the CIS/6-31G(d,p) level, were added step by step until a converged final potential energy surface was obtained. The interpolated potential energy curves started converging to the final potential surface after 9 points were added, but the vibrationally adiabatic energy curves were converged after 15 points were added. This study shows that the MCMM method was applied successfully to make quite reasonable potential and adiabatic energy curves for the excitedstate double proton transfer reaction.

No stable intermediates are present in the potential energy curve along the reaction coordinate of the ESDPT in the 1:1 7-azaindole:water complex, indicating that these two protons are transferred concertedly. This study also shows that two protons move very asynchronously to make an $\mathrm{H}_{3} \mathrm{O}^{+}$-like moiety at the TS.

Acknowledgments. This study was supported by the Kyung
Hee University Research Fund in 2007 (KHU-20070776).

Supplimentary Data. New and modified MM3 parameters are available on request from the corresponding author (Yongho Kim, Fax: 82-31-203-5773, E-mail: yhkim@khu.ac.kr).

\section{References}

1. Taylor, C. A.; El-Bayoumi, M. A.; Kasha, M. Proc. Natl. Acad. Aci. U.S.A. 1969, 63, 253.

2. Chaban, G. M.; Gordon, M. S. J. Phys. Chem. A 1999, 103, 185.

3. Gordon, M. S. J. Phys. Chem. 1996, 100, 3974.

4. Huang, Y.; Arnold, S.; Sulkes, M. J. Phys. Chem. 1996, 100, 4734.

5. Chapman, C. F.; Maroncelli, M. J. Phys. Chem. 1992, 96, 8430.

6. Chen, Y.; Gai, F.; Petrich, J. W. J. Am. Chem. Soc. 1993, 115, 10158.

7. Chou, P.-T.; Martinez, M. L.; Cooper, W. C.; McMorrow, D.; Collins, S. T.; Kasha, M. J. Phys. Chem. 1992, 96, 5203.

8. Kwon, O.-H.; Jang, D.-J. J. Phys. Chem. B 2005, 109, 20479.

9. Kwon, O.-H.; Lee, Y.-S.; Park, H. J.; Kim, Y.; Jang, D.-J. Angew. Chem. 2004, 43, 5792.

10. Mente, S.; Maroncelli, M. J. Phy. Chem. A 1998, 102, 3860.

11. Moog, R. S.; Maroncelli, M. J. Phy. Chem. 1991, 95, 10359.

12. Waluk, J. Acc. Chem. Res. 2003, 36, 832.

13. Chou, P. T.; Yu, W. S.; Wei, C. Y.; Cheng, Y. M.; Yang, C. Y.J. Am. Chem. Soc. 2001, 123, 3599.

14. Hsieh, W.-T.; Hsieh, C.-C.; Lai, C.-H.; Cheng, Y.-M.; Ho, M.-L.; Wang, K. K.; Lee, G.-H.; Chou, P.-T. Chem. Phys. Chem. 2008, 9, 293.

15. Kina, D.; Nakayama, A.; Noro, T.; Taketsugu, T.; Gordon, M. S. J. Phys. Chem. A 2008, 112, 9675.

16. Hung, F.-T.; Hu, W.-P.; Li, T.-H.; Cheng, C.-C.; Chou, P.-T. J. Phys. Chem. A 2003, 107, 3244.

17. Kyrychenko, A.; Waluk, J. J. Phys. Chem. A 2006, 110, 11958.

18. Nam, K.; Kim, Y. J. Chem. Phys. 2009, 130, 144310.

19. Podolyan, Y.; Gorb, L.; Leszczynski, J. J. Phys. Chem. A 2002, $106,12103$.

20. Kim, Y.; Lim, S.; Kim, Y. J. Phys. Chem. A 1999, 103, 6632.

21. Lim, J.-H.; Lee, E. K.; Kim, Y. J. Phys. Chem. A 1997, 101, 2233.

22. Kim, Y.; Corchado, J. C.; Villa, J.; Xing, J.; Truhlar, D. G. J. Chem. Phys. 2000, 112, 2718.

23. Kim, Y. J. Phys. Chem. A 2005, 110, 600.

24. Ischtwan, J.; Collins, M. J. Chem. Phys. 1994, 100, 8080.

25. Nguyen, K. A.; Rossi, I.; Truhlar, D. G. J. Chem. Phys. 1995, 5522.

26. Frisch, M. J.; Trucks, G. W.; Schlegel, H. B.; Scuseria, G. E.; Robb, M. A.; Cheeseman, J. R.; Montgomery, J. A., Jr.; Vreven, T.; Kudin, K. N.; Burant, J. C.; Millam, J. M.; Iyengar, S. S.; Tomasi, J.; Barone, V.; Mennucci, B.; Cossi, M.; Scalmani, G.; Rega, N.; Petersson, G. A.; Nakatsuji, H.; Hada, M.; Ehara, M.; Toyota, K.; Fukuda, R.; Hasegawa, J.; Ishida, M.; Nakajima, T.; Honda, Y.; Kitao, O.; Nakai, H.; Klene, M.; Li, X.; Knox, J. E.; Hratchian, H. P.; Cross, J. B.; Bakken, V.; Adamo, C.; Jaramillo, J.; Gomperts, R.; Stratmann, R. E.; Yazyev, O.; Austin, A. J.; Cammi, R.; Pomelli, C.; Ochterski, J. W.; Ayala, P. Y.; Morokuma, K.; Voth, G. A.; Salvador, P.; Dannenberg, J. J.; Zakrzewski, V. G.; Dapprich, S.; Daniels, A. D.; Strain, M. C.; Farkas, O.; Malick, D. K.; Rabuck, A. D.; Raghavachari, K.; Foresman, J. B.; Ortiz, J. V.; Cui, Q.; Baboul, A. G.; Clifford, S.; Cioslowski, J.; Stefanov, B. B.; Liu, G.; Liashenko, A.; Piskorz, P.; Komaromi, I.; Martin, R. L.; Fox, D. J.; Keith, T.; Al-Laham, M. A.; Peng, C. Y.; Nanayakkara, A.; Challacombe, M.; Gill, P. M. W.; Johnson, B.; Chen, W.; Wong, M. W.; Gonzalez, C.; Pople, J. A. Gaussian 03, Gaussian, Inc.: Wallingford, CT, 2004

27. Garrett, B. C.; Truhlar, D. G.; Grev, R. S.; Magnuson, A. W. J. Phys. Chem. 1980, 84, 1730.

28. Truhlar, D. G.; Isaacson, A. D.; Garrett, B. C. In Theory of Chemical Reaction Dynamics; Baer, M., Ed.; CRC Press: Boca Raton, 
FL, 1985; Vol. 4, p 65.

29. Liu, Y.-P.; Lynch, G. C.; Truong, T. N.; Lu, D.-H.; Truhlar, D. G.; Garrett, B. C. J. Am. Chem. Soc. 1993, 115, 2408.

30. Skodje, R. T.; Truhlar, D. G.; Garrett, B. C. J. Phys. Chem. 1981, 85,3019 .

31. Garrett, B. C.; Joseph, T.; Truong, T. N.; Truhlar, D. G. Chem. Phys. 1989, 136, 271.

32. Fernandez-Ramos, A.; Truhlar, D. G. J. Chem. Phys. 2001, 114, 1491.

33. Corchado, J. C.; Chuang, Y.-Y.; Fast, P. L.; Villa, J.; W.-P. Hu; Liu, Y.-P.; Lynch, G. C.; Nguyen, A.; Jackels, C. F.; V. S. Melissas; Lynch, B. J.; Rossi, I.; Coitino, E. L.; Fernandez-Ramos, A.; Pu, J.; Steckler, R.; Garrett, B. C.; Isaacson, A. D.; Truhlar, D. G. POLY-
RATE 8.7.2, University of Minnesota, Minneapolis, 2002.

34. Albu, T. V.; Corchado, J. C.; Kim, Y.; Villa, J.; Xing, J.; Truhlar, D. G. MC-TINKERATE 8.8, University of Minnesota, Minneapolis, 2002.

35. Corchado, J. C.; Chuang, Y.-Y.; Fast, P. L.; Hu, W.-P.; Liu, Y.-P.; Lynch, G. C.; Nguyen, K. A.; Jackels, C. F.; Ramos, A. F.; Ellingson, B. A.; Lynch, B. J.; Zheng, J.; Melissas, V. S.; Villa, J.; Rossi, I.; Coitino, E. L.; Pu, J.; Albu, T. V.; Steckler, R.; Garrett, B. C.; Isaacson, A. D.; Truhlar, D. G. Polyrate-version 9.7, University of Minnesota, Minneapolis, U.S.A., 2007

36. Albu, T. V.; Corchado, J. C.; Kim, Y.; Villa, J.; Xing, J.; Truhlar, D. G. MC-TINKER 1.0, University of Minnesota, Minneapolis, 2002 37. Sakota, K.; Sekiya, H. J. Phys. Chem. A 2009, 113, 2663. 